\title{
The Health Humanities and Emergency Medical Services (EMS): A Call to Action
}

\author{
Kiriana A. Morse, MD; $\odot$ Kamna S. Balhara, MD, MA; Nathan A. Irvin, MD; Matthew J. Levy, DO, MSc $\odot$
}

Department of Emergency Medicine, Johns Hopkins University School of Medicine, Baltimore, Maryland USA

\section{Correspondence:}

Kiriana Morse, MD

PGY-3 Emergency Medicine Resident

Johns Hopkins University School of Medicine Department of Emergency Medicine 1830 E. Monument St, Suite 6-100 Baltimore, Maryland 21287 USA

E-mail: kmorse7@jhmi.edu

Conflicts of interest: The authors declare none.

Keywords: continuing education; Emergency Medical Services; humanities

Abbreviation:

EMS: Emergency Medical Services

Received: November 9, 2021

Accepted: December 5, 2021

doi:10.1017/S1049023X22000243

(C) The Author(s), 2022. Published by

Cambridge University Press on behalf of the

World Association for Disaster and Emergency Medicine.

\begin{abstract}
In the context of an on-going global pandemic that has demanded increasingly more of our Emergency Medical Services (EMS) clinicians, the health humanities can function to aid in educational training, promoting resilience and wellness, and allowing opportunity for selfexpression to help prevent vicarious trauma.

As the social, cultural, and political landscape of the United States continues to require an expanded scope of practice from our EMS clinicians, it is critical that the health humanities are implemented as not only part of EMS training, but also as part of continued practice in order to ensure the highest quality patient-centered care while protecting the longevity and resilience of EMS clinicians.
\end{abstract}

Morse KA, Balhara KS, Irvin NA, Levy MJ. The health humanities and Emergency Medical Services (EMS): a call to action. Prehosp Disaster Med. 2022;37(2):150-151.

Emergency Medical Services (EMS) clinicians are often a patient's first point of contact with health care systems that have long been plagued by disparities and inequities. Prehospital personnel witness firsthand the impacts of health inequity and are frequently exposed to circumstances that may contribute to moral injury and burnout. ${ }^{1,2}$ Recent stressors, catalyzed by the COVID-19 pandemic, have further exacerbated and illuminated the multitude of threats to clinicians' well-being. ${ }^{3}$ Increasingly, EMS clinicians are being called upon to serve in expanded roles in the care of patients beyond conventional emergency care settings, a task that, to be successful, requires a fundamentally different and nuanced approach. ${ }^{4,5} \mathrm{We}$ find ourselves in a pivotal moment of this crisis, one which demands a unique and actionable solution. Systemic and societal changes are needed to enact sustained solutions to these issues, and no single approach will offer a panacea. We suggest looking beyond the traditional paradigms of health professions education to other fields, specifically the health humanities, to help equip EMS clinicians with the knowledge, skills, and attributes needed to advance health equity while safeguarding their own well-being.

The health humanities have gained increasing attention in recent decades. Situated at the intersection of health with the social sciences, humanities, and arts, the health humanities represent a broad and inclusive approach to both understanding and transforming the experiences of health, illness, and the provision of care. ${ }^{6}$ Recognizing that medicine itself is only a minor contributor to overall health, the health humanities seek to study the social, political, cultural, and historical factors that impact health and health care. Moving beyond the physician-centric vision of narrower fields such as the medical humanities, the health humanities also center those voices often relegated to the periphery of conversations about health, including the voices of patients, allied health professionals, and their caregivers. The health humanities provide an interdisciplinary scaffold for collectively assessing, critiquing, and improving structural inequities in medicine, while providing avenues for clinicians to reflect upon and process the individual challenges they encounter in their practice.

Arts- and humanities-based approaches have gained an important foothold in medical education, with both the Association of American Medical Colleges (Washington, DC USA) and the National Academies of Sciences, Engineering, and Medicine (Washington, DC USA) deeming the arts and humanities as fundamental to medical education across the spectrum, from pre-health undergraduate students to physicians in practice. $^{7}$ Existing evidence on the use of humanities-based approaches in health professions education suggests that the arts and humanities function to: (1) improve the mastery of important clinical skills; (2) enhance clinicians' ability to take and share perspectives; (3) create avenues for introspection and resilience; and (4) encourage advocacy and critical reflection on sociocultural issues impacting health. ${ }^{8}$ 
Though such impacts may be of relevance to all health care professionals, the majority of humanities-based interventions have targeted physicians-in-training, with a paucity of opportunities for EMS trainees or clinicians. ${ }^{9}$ Many are advocating that we transition away from the historical phrasing of "medical-humanities" towards health humanities, shifting the dynamic from a purely medical focus to a multifaceted and interdisciplinary approach that incorporates the many other voices engaged in health and health care that face the same issues yet receive little attention. ${ }^{10,11}$ This collaborative and inclusive approach is more aptly reflective of the true interdisciplinary nature of our everyday health care teams. At this crucial juncture, EMS clinicians represent a critical and under-represented voice in this dialogue.

The practice of EMS is uniquely impacted by the social, political, and cultural factors that shape personal and population health. Essentially, EMS clinicians have the truly distinctive vantage point of meeting patients in the highly personal settings of their homes, places of work, and recreation, and are well-positioned to identify the social determinants of health that may affect each individual or community. They are also often intimate witnesses to the harms of social injustice and structural racism. Further, EMS clinicians often navigate emotionally charged and complex circumstances and often need to make rapid decisions and interventions with ambiguous or limited information. These EMS clinicians, as true frontline workers critical to the functioning of the United States health care system, stand to both gain from and contribute to the potential benefits of the health humanities and represent integral stakeholders that should be included in the discussion.

In the United States, the National EMS Scope of Practice Model and the National EMS Educational Standards make generic reference to the inclusion of the psychosocial impact related to emergency and nonemergency conditions as related to EMS education. ${ }^{12,13}$ Opportunity exists to enhance and expand upon this by bolstering the inclusion of health humanities in EMS education. Doing such has multiple benefits, as the incorporation of historical, social, political, and environmental context inherent to the health humanities leads to a nuanced understanding of the structural and social determinants of health, which help to contextualize individual illness and community health, and will help EMS clinicians navigate their expanding role. Further, EMS clinicians often bear witness to gruesome and traumatic events, and then must rapidly move on to their next patient or task. Dedicated time and means for self-expression, reflection, and communication through the lenses of the humanities can create space to process these scenes, begin healing, and perhaps even limit vicarious trauma. Exposure to the humanities may also help enhance both empathy and tolerance of uncertainty, attributes tied to improved patient care, patient satisfaction, and clinician well-being. ${ }^{14-18}$

As the gaps in our health care system are continually exposed, both during this pandemic and in the years to come, we are charged with providing the highest quality patient-centered care while protecting the longevity and resilience of EMS clinicians. This charge is foundational to the functioning of our health care systems and, as increasing attention is brought to bear on the humanities and its integration is expanded beyond physician-centric models, we must ensure that EMS clinicians have a permanent seat at the table. All EMS trainees and clinicians should have the opportunity to both engage with and lead innovation within the health humanities for the sake of themselves and the patients they will continue to treat.
References

1. ALmutairi MN, E1 Mahalli AA. Burnout and coping methods among Emergency Medical Services professionals. J Multidiscip Healthc. 2020;13:271-279.

2. Mountfort S, Wilson J. EMS Provider Health and Wellness. Treasure Island, Florida USA: StatPearls Publishing; 2021.

3. Tan BYQ, Kanneganti A, Lim LJH, et al. Burnout and associated factors among health care workers in Singapore during the COVID-19 pandemic. J Am Med Dir Assoc. 2020;21(12):1751-1758

4. Plat FM, Peters YAS, Loots FJ, et al. Ambulance dispatch versus general practitioner home visit for highly urgent out-of-hours primary care. Fam Pract. 2018;35(4):440 445

5. Oosterwold J, Sagel D, Berben S, Roodbol P, Broekhuis M. Factors influencing the decision to convey or not to convey elderly people to the emergency department after emergency ambulance attendance: a systematic mixed studies review. BMJ Open. 2018;8(8): 021732

6. Crawford P, Brown B, Baker C, Tischler V, Abrams B. Health Humanities. London, England: Palgrave-Macmillan; 2015.

7. National Academies of Sciences, Engineering, and Medicine. The Integration of the Humanities and Arts with Sciences, Engineering, and Medicine in Higher Education: Branches from the Same Tree. Washington, DC USA: National Academies Press; 2018.

8. Moniz T, Golafshani M, Gaspar CM, et al. The prism model: advancing a theory of practice for arts and humanities in medical education. Perspect Med Educ. 2021;10(4):207-214.
9. Howley L, Gaufberg E, King B. The Fundamental Role of the Arts and Humanities in Medical Education. Washington, DC USA: AAMC; 2020.

10. Jones T, Blackie M, Garden R, Wear D. The almost right word: the move from medical to health humanities. Acad Med. 2017;92(7):932-935.

11. Sklar DP. Health humanities and medical education: joined by a common purpose. Acad Med. 2017;92(12):1647-1649.

12. The National Highway Traffic Safety Administration. National Emergency Medical Services Education Standards; 2009. https://www.ems.gov/pdf/National-EMSEducation-Standards-FINAL-Jan-2009.pdf. Accessed November 2021.

13. National Association of State EMS Officials. National EMS Scope of Practice Model 2019 (Report No. DOT HS 812-666). Washington, DC USA: National Highway Traffic Safety Administration; 2019.

14. Wolfshohl JA, Bradley K, Bell C, et al. Association between empathy and burnout among emergency medicine physicians. J Clin Med Res. 2019;11(7):532-538.

15. Strout TD, Hillen M, Gutheil C, et al. Tolerance of uncertainty: a systematic review of health and health care-related outcomes. Patient Educ Couns. 2018;101(9):1518-1537.

16. Wang H, Kline JA, Jackson BE, et al. Association between emergency physician selfreported empathy and patient satisfaction. PLoS One. 2018;13(9):e0204113.

17. Bentwich ME, Gilbey P. More than visual literacy: art and the enhancement of tolerance for ambiguity and empathy. BMC Med Educ. 2017;17(1):200.

18. Klugman CM, Lamb EG, (Eds). Research Methods in Health Humanities. Oxford, UK: Oxford University Press; 2019. 